\title{
Laser-induced transitions between triply excited hollow states
}

\author{
L. B. Madsen, ${ }^{1, *}$ P. Schlagheck, ${ }^{1,2}$ and P. Lambropoulos ${ }^{1,3}$ \\ ${ }^{1}$ Max-Planck-Institut für Quantenoptik, Hans-Kopfermann-Straße 1, D-85748 Garching, Germany \\ ${ }^{2}$ Laboratoire de Physique Théorique et Modèles Statistiques, Bâtiment 100, F-91405 Orsay Cedex, France \\ ${ }^{3}$ Foundation for Research and Technology Hellas, Institute of Electronic Structure and Laser, P.O. Box 1527, \\ Heraklion 71110 Crete, Greece
}

(Received 16 June 2000; published 14 November 2000)

\begin{abstract}
Using complex scaling and a correlated basis constructed in terms of $B$ splines, we calculate the $\mathrm{Li}^{+}$ photoion yield in the presence of a laser-induced coupling between the triply excited $2 s^{2} 2 p\left({ }^{2} P^{o}\right)$ and $2 s 2 p^{2}\left({ }^{2} D^{e}\right)$ resonances in lithium, the first of which is assumed to be excited by synchrotron radiation from the ground state. The laser coupling between the triply excited states is shown to lead to a significant and readily measurable modification of the line profile which provides a unique probe of the dipole strength between highly correlated triply excited states. We also present results for some higher-lying triply excited states of ${ }^{2} P^{o}$ symmetry.
\end{abstract}

PACS number(s): 32.80.Hd, 32.80.Dz, 32.80.Fb, 32.80.Qk

\section{INTRODUCTION}

A triply excited state in lithium, also referred to as a hollow state, represents a fundamental case of highly correlated three-electron dynamics in a purely Coulombic four-body system, as attested to by the continuing avalanche of related papers $[1-23]$ over the last six years. Photoexcitation by synchrotron radiation has proven to be a very successful method to study energies and widths of such states. They were studied by photoabsorption [1], photoion [2-4], and photoelectron measurements [5-10], and an impressive number of triply excited states was reported. States with empty $K$ and $L$ shells were observed [9,4], and several Rydberg series identified $[11-15,24]$. The results were compared with $R$-matrix $[5-10,12,13]$ and Dirac-Fock calculations $[3,4]$, and the agreement is generally good. There is also excellent agreement between experimental values and very accurate calculations using the saddle-point complex rotation method $[17,18,9]$. In addition, the hyperspherical coordinate approach was used to analyze and visualize the electron correlations, in an effort to obtain a classification in terms of approximate quantum numbers [19-23].

The selectivity of the photoexcitation technique means that only states of ${ }^{2} P^{o}$ symmetry can be reached from the ground state. It is, however, equally interesting to investigate states of different symmetry and parity, as in one experiment [7], where a laser excited the $1 s^{2} 2 p\left({ }^{2} P^{o}\right)$ state from which synchrotron radiation produced even-parity triply excited states. This is one way of reaching hollow even-parity states which still probes their connection with bound states of the neutral. However, until very recently [16] nothing was known about the fundamental interaction of the triply excited states themselves with radiation. In particular, the induced coupling between such highly correlated states, which provides a much more detailed probe of three-electron correlation than the excitation from bound states only, was not ex-

\footnotetext{
*Present address: Institute of Physics and Astronomy, University of Aarhus, DK-8000 Aarhus C, Denmark.
}

amined. In Ref. [16] we undertook such an investigation, and presented a report on the generic effect of photoabsorption from the $1 s^{2} 2 s\left({ }^{2} S^{e}\right) \mathrm{Li}$ ground state to the $2 s^{2} 2 p\left({ }^{2} P^{o}\right)$ triply excited state, when the system is embedded in a judiciously chosen laser field. Clearly, this allows one to investigate states of ${ }^{2} S^{e}$ and ${ }^{2} D^{e}$ symmetry. Specifically, we consider laser coupling between $2 s^{2} 2 p\left({ }^{2} P^{o}\right)$ and $2 s 2 p^{2}\left({ }^{2} D^{e}\right)$ triply excited states, which is on resonance at a near-optical wavelength corresponding to a photon energy of $\simeq 2.5 \mathrm{eV}$. Such a coherent coupling leads to a significant modification of the line profile of the $\mathrm{Li}^{+}$photoion spectrum. At a sufficiently high, but still modest, laser intensity, the spectrum develops an ac Stark splitting which is directly related to and provides a measure of the dipole matrix element between the triply excited states. In this paper, we present a much more detailed account of the theoretical model, the atomic structure calculations as well as of the calculation of the atomic parameters.

It should be noted that the processes we examine require an analysis of the time development of the system driven by the radiation. Despite the existence of a number of successful approaches $[1-15,17-25]$ to the calculation of the atomic structure of three-electron systems, our needs in this work necessitate an approach, perhaps more demanding for accurate structure, but more convenient for the study of dynamics. Although some of the results on line-shape modification in this paper could be obtained through rate equations, in view of rapid developments in pulsed, bright, shortwavelength sources such as the free-electron laser, it should be feasible in the near future to explore the full range of dynamical effects. Hence we present a theoretical formalism capable of handling this situation.

This paper is organized as follow: In Sec. II, we present a theoretical model describing the coherent coupling of two autoionizing triply excited states. This section also includes an analysis of an analytical formula which, in the weakprobe limit, corresponding to a relatively weak coupling by the synchrotron pulse between the ground state and the lower triply excited state, accounts very well for the results obtained by a direct numerical integration of the equations of motions describing the dynamics of the system. In Sec. III- 


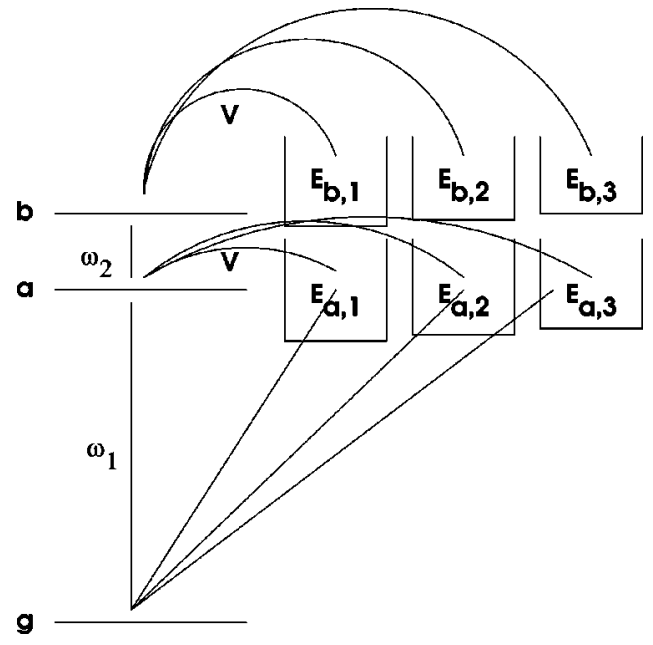

FIG. 1. The level scheme under consideration. The $1 s^{2} 2 s\left({ }^{2} S^{e}\right)$ Li ground state is denoted by $|g\rangle$. The synchrotron coupling $|g\rangle$ with the discrete part $|a\rangle$ and the continuum parts $\left|E_{a, j}\right\rangle$ (j $=1,2,3, \ldots)$ of the lower triply excited state $2 s^{2} 2 p^{2} P^{o}$ has an angular frequency $\omega_{1}$. The laser coupling the $2 s^{2} 2 p\left({ }^{2} P^{o}\right)$ and $2 s 2 p^{2}\left({ }^{2} D^{e}\right)$ triply excited states, the latter of which has a discrete part denoted by $|b\rangle$ and continuum parts denoted by $\left|E_{b, k}\right\rangle$ ( $k$ $=1,2,3, \ldots)$, has an angular frequency $\omega_{2}$. The discrete parts $|a\rangle$ and $|b\rangle$ are partly coupled to the continua via the configuration interaction $V$, giving rise to the autoionization widths $\Gamma_{a}$ and $\Gamma_{b}$, and partly via laser-induced ionization described by the widths $\gamma_{a}$ and $\gamma_{b}$. Only three out of the infinitely many continua coupled to $|a\rangle$ and $|b\rangle$, respectively, are shown in the figure.

we describe the theoretical calculations. Section III A describes how the atomic structure is calculated within a fully correlated basis, and how the positions and widths of the triply excited states are determined by complex scaling. In Sec. III B we calculate the atomic parameters entering the model. In Sec. III C we present results on energy positions and widths for some higher-lying triply excited states of ${ }^{2} P^{o}$ symmetry, and compare our values with existing data. Additionally, we calculate the light-induced coupling between the ground state and the $3 s^{2} 3 p\left({ }^{2} P^{o}\right)$ state, the lowest triply excited state with empty $K$ and $L$ shells. Explicit formal expressions of the parameters as well as further discussion of these are found in Appendices A and B. With the parameters at hand, the model produces the results on laser-induced transitions between triply excited states discussed in Sec. IV.

\section{MODEL}

To introduce the basic idea and scheme, let $|g\rangle$ be the $1 s^{2} 2 s\left({ }^{2} S^{e}\right)$ ground state and $|a\rangle$ and $|b\rangle$ the discrete parts belonging to the $2 s^{2} 2 p\left({ }^{2} P^{o}\right)$ and $2 s 2 p^{2}\left({ }^{2} D^{e}\right)$ triply excited states, respectively. The triply excited states $2 s^{2} 2 p\left({ }^{2} P^{o}\right)$ and $2 s 2 p^{2}\left({ }^{2} D^{e}\right)$ are both energetically above the $\mathrm{Li}^{2+}$ ionization threshold. A multitude of channels is therefore open for autoionization. Let $|a\rangle$ and $|b\rangle$ autoionize into the continua $\left|E_{a, j}\right\rangle(j=1,2,3, \ldots)$ and $\left|E_{b, k}\right\rangle(k=1,2,3, \ldots)$, respectively. The indices of these states denote the continua corresponding to different ionization thresholds. Figure 1 shows the basic level scheme. Only three continua for each auto- ionizing state are shown. The figure shows some of the couplings entering into this problem. The $2 s^{2} 2 p\left({ }^{2} P^{o}\right)$ state, with a discrete part $|a\rangle$, is assumed to be excited by a synchrotron pulse of frequency $\omega_{1} \simeq 142.3 \mathrm{eV}$ in the presence of a laser of frequency $\omega_{2}$ and a pulse duration preferably longer than that of the synchrotron [atomic units $(\hbar=m=e$ $=1$ ) are used throughout unless otherwise indicated]. Both radiation sources are assumed to be linearly polarized along the same direction. The frequency $\omega_{2}$ is chosen so as to be tunable around resonance with the transition $2 s^{2} 2 p\left({ }^{2} P^{o}\right)$ $\rightarrow 2 s 2 p^{2}\left({ }^{2} D^{e}\right)$, which means that $\omega_{2} \simeq 2.5 \mathrm{eV}$. Note that the excited states $1 s^{2} 2 p\left({ }^{2} P^{o}\right)$ and $1 s^{2} 3 p\left({ }^{2} P^{o}\right)$ of $\mathrm{Li}$ are located about 1.85 and $3.8 \mathrm{eV}$, respectively, above $|g\rangle$, thus being far from resonance with $\omega_{2}$. The transition induced by the relatively weak radiation source operated at $\omega_{1}$ can be described by a single rate, but the transition by the much stronger source operated at $\omega_{2}$ cannot be so, thus requiring a strong (nonperturbative) coupling of the triply excited states. Note that the continua $\left|E_{a, j}\right\rangle$ are accessed not only from the discrete part $|a\rangle$ (due to the configuration interaction) but also directly by photoionization from the ground state. Given the short duration of both radiation sources involved, and the fast autoionization of the resonances, spontaneous radiative decay may be safely ignored, and the problem is conveniently formulated in terms of the time-dependent Schrödinger equation. Note that spontaneous emission will eventually become important as we move along the $\mathrm{Li}$ isoelectronic sequence. This is because the spontaneous emission rate increases with the nuclear charge $Z$, due to an increase in the free-space density of modes of the electromagnetic vacuum field (which more than compensates for the decrease of the dipole moment), whereas the autoionization width of the triply excited $2 s^{2} 2 p$ (and $2 s 2 p^{2}$ ) state decreases along the isoelectronic sequence [26].

The wave function of the atom under the fields is expressed as

$$
\begin{aligned}
|\Psi(t)\rangle= & C_{g}(t)|g\rangle+C_{a}(t)|a\rangle+C_{b}(t)|b\rangle \\
& +\sum_{j} \int d E_{a, j} C_{E_{a, j}}(t)\left|E_{a, j}\right\rangle \\
& +\sum_{k} \int d E_{b, k} C_{E_{b, k}}(t)\left|E_{b, k}\right\rangle .
\end{aligned}
$$

This expression is substituted into the time-dependent Schrödinger equation

$$
i \partial_{t}|\Psi(t)\rangle=H|\Psi(t)\rangle,
$$

where $H$ is written as $H=H_{0}+V+D$, with $H_{0}|s\rangle$ $=E_{s}|s\rangle \quad\left(s=g, a, b, E_{a, j}, E_{b, k}\right), V$ being the configuration interaction coupling the discrete parts of the triply excited states to the continua, and $D$ the dipole interaction. Projection on the individual states in the expansion of Eq. (1) of $|\Psi(t)\rangle$ leads to a set of coupled differential equations containing amplitudes for the discrete parts as well as for the continua, also including laser-induced continuum-continuum couplings. Ignoring the latter is usually a well-justified approximation at the intensities we need consider. We intro- 
duce the slowly varying amplitudes $c_{g}(t)=C_{g}(t) e^{i E_{g} t}$, $c_{a}(t)=C_{a}(t) e^{i\left(E_{g}+\omega_{1}\right) t}, c_{b}(t)=C_{b}(t) e^{i\left(E_{g}+\omega_{1}+\omega_{2}\right) t}, c_{E_{a, j}}(t)$ $=C_{E_{a, j}}(t) e^{i\left(E_{g}+\omega_{1}\right) t}$, and $c_{E_{b, k}}(t)=C_{E_{b, k}}(t) e^{i\left(E_{g}+\omega_{1}+\omega_{2}\right) t}$, and keep only the time-dependent exponentials which are resonant with the photon frequencies (the rotating-wave approximation). This amounts to neglecting virtual photon exchange processes. As the final step, we eliminate the continua adiabatically (which basically means that they are treated as sinks) by setting the time derivative of the slowly varying continuum amplitudes equal to zero, $\dot{c}_{E}(t)=0$. We arrive at the following set of equations for the timedependent discrete-state amplitudes:

$$
\begin{aligned}
& i \partial_{t} c_{g}=\left[\sum_{j} \int d E_{a, j} \frac{\left|\Omega_{g E_{a, j}}(t)\right|^{2}}{E_{g}+\omega_{1}-E_{a, j}}\right] c_{g} \\
& +\left[\Omega_{g a}(t)+\sum_{j} \int d E_{a, j} \frac{\Omega_{g E_{a, j}}(t) V_{a E_{a, j}}}{E_{g}+\omega_{1}-E_{a, j}}\right] c_{a} \\
& +\left[\sum_{j} \int d E_{a, j} \frac{\Omega_{g E_{a, j}}(t) \Omega_{E_{a, j} b}(t)}{E_{g}+\omega_{1}-E_{a, j}}\right] c_{b}, \\
& i \partial_{t} c_{a}=\left[\Omega_{a g}(t)+\sum_{j} \int d E_{a, j} \frac{V_{a E_{a, j}} \Omega_{E_{a, j} g}(t)}{E_{g}+\omega_{1}-E_{a, j}}\right] c_{g} \\
& +\left[E_{a}-E_{g}-\omega_{1}+\sum_{j} \int d E_{a, j} \frac{\left|V_{a, E_{a, j}}\right|^{2}}{E_{g}+\omega_{1}-E_{a, j}}\right. \\
& \left.+\sum_{k} \int d E_{b, k} \frac{\left|\Omega_{a E_{b, k}}(t)\right|^{2}}{E_{g}+\omega_{1}+\omega_{2}-E_{b, k}}\right] c_{a} \\
& +\left[\Omega_{a b}(t)+\sum_{j} \int d E_{a, j} \frac{V_{a E_{a, j}} \Omega_{E_{a, j} b}(t)}{E_{g}+\omega_{1}-E_{a, j}}\right. \\
& \left.+\sum_{k} \int d E_{b, k} \frac{\Omega_{a E_{b, k}}(t) V_{E_{b, k} b}}{E_{g}+\omega_{1}+\omega_{2}-E_{b, k}}\right] c_{b}, \\
& i \partial_{t} c_{b}=\left[\sum_{j} \int d E_{a, j} \frac{\Omega_{b E_{a, j}}(t) \Omega_{E_{a, j} g}(t)}{E_{g}+\omega_{1}-E_{a, j}}\right] c_{g} \\
& +\left[\Omega_{b a}(t)+\sum_{j} \int d E_{a, j} \frac{\Omega_{b E_{a, j}(t) V_{E_{a, j} a}}}{E_{g}+\omega_{1}-E_{a, j}}\right. \\
& \left.+\sum_{k} \int d E_{a, k} \frac{V_{b E_{b, k}} \Omega_{E_{b, k} a}(t)}{E_{g}+\omega_{1}+\omega_{2}-E_{b, k}}\right] c_{a} \\
& +\left[E_{b}-E_{g}-\omega_{1}-\omega_{2}+\sum_{j} \int d E_{a, j} \frac{\left|\Omega_{b E_{b, k}}(t)\right|^{2}}{E_{g}+\omega_{1}-E_{a, j}}\right. \\
& \left.+\sum_{k} \int d E_{b, k} \frac{\left|V_{b E_{b, k}}\right|^{2}}{E_{g}+\omega_{1}+\omega_{2}-E_{b, k}}\right] c_{b},
\end{aligned}
$$

with $V_{a E_{a, j}}$ and $V_{b E_{b, k}}$ the configuration interactions of the discrete parts $(|a\rangle,|b\rangle)$ with the continua $\left(\left|E_{a, j}\right\rangle,\left|E_{b, k}\right\rangle\right)$, and $\Omega_{g a}(t)=\frac{1}{2} D_{g a, z} F_{1}(t)$, and $\Omega_{a b}(t)=\frac{1}{2} D_{a b, z} F_{2}(t)$ the Rabi frequencies between $|g\rangle \rightarrow|a\rangle$, and $|a\rangle \rightarrow|b\rangle$, respectively. Here $D_{g a, z}$ and $D_{a b, z}$ are the corresponding dipole matrix elements, along the linear polarization direction of the laser, $\hat{z}$, and $F_{i}(t) \quad(i=1$ and 2$)$ are the time-dependent pulses of the synchrotron and the assisting laser field. The integration over the continua, in Eq. (3), runs from the lowest threshold of the particular continuous spectrum. The evaluation of the atomic coupling parameters entering the above set of equations is significantly simplified by using the following identity for generalized functions (distributions):

$$
\lim _{\eta \rightarrow 0_{+}} \frac{1}{E_{0}-E+i \eta}=\mathrm{P} \frac{1}{E_{0}-E}-i \pi \delta\left(E_{0}-E\right),
$$

where $\mathrm{P}$ denotes the principal value part, and $\delta$ is the Dirac delta function. Repetitive use of Eq. (4) in Eq. (3) leads to

$$
\begin{aligned}
& i \partial_{t} c_{g}=\left[S_{g}-\frac{i}{2} \gamma_{g}\right] c_{g}+\widetilde{\Omega}_{g a}\left(1-\frac{i}{q_{a}}\right) c_{a}+\left[S_{g b}-\frac{i}{2} \gamma_{g b}\right] c_{b}, \\
& i \partial_{t} c_{a}=\tilde{\Omega}_{a g}\left(1-\frac{i}{q_{a}}\right) c_{g}-\left[\delta_{1}+\frac{i}{2}\left(\Gamma_{a}+\gamma_{a}\right)\right] c_{a} \\
& +\tilde{\Omega}_{a b}\left(1-\frac{i}{q_{a b}}\right) c_{b}, \\
& i \partial_{t} c_{b}=\left[S_{b g}-\frac{i}{2} \gamma_{b g}\right] c_{g}+\tilde{\Omega}_{b a}\left(1-\frac{i}{q_{a b}}\right) c_{a}-\left[\delta_{1}+\delta_{2}\right. \\
& \left.+\frac{i}{2}\left(\Gamma_{b}+\gamma_{b}\right)\right] c_{b}
\end{aligned}
$$

with

$$
\begin{aligned}
& \delta_{1}=\omega_{1}-\left(E_{a}^{r}+S_{a}-E_{g}-S_{g}\right), \\
& \delta_{2}=\omega_{2}-\left(E_{b}^{r}+S_{b}-E_{a}^{r}-S_{a}\right)
\end{aligned}
$$

the detunings, $E_{i}^{r}$ and $\Gamma_{i}(i=a, b)$ the resonance energies and widths of the triply excited states, $S_{g}, S_{a}, S_{b}, S_{g b}$ and $\gamma_{g}, \gamma_{a}, \gamma_{b}, \gamma_{g b}$ the laser-induced shifts and widths, $\tilde{\Omega}_{g a}$ and $\widetilde{\Omega}_{a b}$ generalized Rabi frequencies including a light-induced shift, and $q_{a}$ and $q_{a b}$ the Fano line-shape parameter for the $|g\rangle-|a\rangle$ transition [27] and its generalization for the $|a\rangle-|b\rangle$ transition. Explicit formal expressions for the parameters of Eq. (5) are given in Appendix A, and Appendix B discusses how to calculate these using complex scaling.

The dominant autoionization decay channels for $|a\rangle$ $=2 s^{2} 2 p\left({ }^{2} P^{o}\right)$ and $|b\rangle=2 s 2 p^{2}\left({ }^{2} D^{e}\right)$ were given by Chung in Ref. [28], and are of the types $1 s 2 p\left({ }^{3,1} P\right)+\epsilon l \quad(l=s, d)$ and $1 s 2 s\left({ }^{3,1} S\right)+\epsilon p$ for $|a\rangle$ and $1 s 2 p\left({ }^{3,1} P\right)+\epsilon l \quad(l=p, f)$ and $1 s 2 s\left({ }^{3,1}\right)+\epsilon d$ for $|b\rangle$. The laser-induced couplings between the discrete state $|a\rangle(|b\rangle)$ and the nonresonant parts of the continua $\left|E_{b, k}\right\rangle, \quad k=1,2,3, \ldots \quad\left(\left|E_{a, j}\right\rangle, j\right.$ $=1,2,3, \ldots)$ are thus expected to be very small, since the dominant configurations do not share common orbitals. 
Thus, to an excellent approximation, we can neglect the ionization widths $\gamma_{a}$ and $\gamma_{b}$ to these nonresonant background parts. We can also neglect $\gamma_{g b}$ and $S_{g b}$, which describe a width and a shift due to the laser-induced coupling from $|g\rangle$ to $|b\rangle$ via the nonresonant part of the continua, $\left|E_{a, j}\right\rangle$. The ac Stark shift of the ground state $S_{g}$ is vanishing at the intensities considered for the short-wavelength source. The laserinduced shifts of the triply excited states $S_{a}$ and $S_{b}$ are ponderomotive in character, and they will consequently be equal to an excellent approximation. Accordingly, the two laserinduced shifts cancel in the detuning, $\delta_{2}$. Having said this, we note that for the photon energies and intensities considered in the present work the shifts $S_{a}$ and $S_{b}$ are negligible.

Equations (5) are readily solved once the atomic parameters are known. The way to calculate the parameters is discussed in Sec. III B and Appendix B. The probability for autoionization or laser-induced ionization into the dominant $\mathrm{Li}^{+}$-ion channel is then calculated as $P_{\text {ion }}=1-\left|c_{g}(T)\right|^{2}$, where $T$ is the pulse duration of the synchrotron radiation. We note in passing that, as the photon energy of the light source responsible for the initial excitation step increases, the probability for ionization into the $\mathrm{Li}^{2+}$ ion channel increases as additional channels corresponding to two-step double autoionization via doubly excited states in the $\mathrm{Li}^{+}$ion begin to compete with the weak direct one-photon two-electron ejection process [see Ref. [29] for a discussion of this resonant two-step double autoionization process in the context of enhanced double ionization in Ba and Refs. $[9,11,8]$ for experiments exploiting that the (high-energy) Auger electron from the doubly excited $\mathrm{Li}^{+}$state to the $\mathrm{Li}^{2+}$ ground state is easily detected].

Although the results to be presented in Sec. IV were obtained by numerical integration of Eq. (5), it is interesting to note that an analytical formula for the photoion rate $R$, can be derived in the weak-probe limit $\left(\widetilde{\Omega}_{g a} \ll 1\right)$

$$
\begin{aligned}
R \simeq & \gamma_{g}+\frac{2 \widetilde{\Omega}_{g a}^{2}}{\left|f\left(\delta_{1}, \delta_{2}\right)\right|^{2}} \\
& \times \operatorname{Re}\left[i\left(1-\frac{i}{q_{a}}\right)^{2}\left(\delta+\frac{i}{2} \Gamma_{b}\right) f^{*}\left(\delta_{1}, \delta_{2}\right)\right],
\end{aligned}
$$

with $\quad f\left(\delta_{1}, \delta_{2}\right)=\left[\delta+(i / 2) \Gamma_{b}\right]\left[\delta_{1}+(i / 2) \Gamma_{a}\right]-\widetilde{\Omega}_{a b}^{2}[1$ $\left.-\left(i / q_{a b}\right)\right]^{2}$ and $\delta=\delta_{1}+\delta_{2}$. The rate is derived from Eq. (5) by assuming $c_{g} \simeq 1$, i.e., from $R=-2 \operatorname{Re}\left[\partial_{t} c_{g}\right]$ and by solving for $c_{a}$ from Eqs. (5b) and (5c) in the steady state. This method is equivalent to performing the derivation with the Laplace transform method in the pole approximation, and the results are identical. If we use $q_{a b} \gg 1$, the expression for $R$ reduces to

$$
\begin{aligned}
R \simeq & \gamma_{g}+\widetilde{\Omega}_{g a}^{2}\left[\left(1-\frac{1}{q_{a}^{2}}\right)\left(\delta^{2} \Gamma_{a}+\frac{\Gamma_{a} \Gamma_{b}^{2}}{4}+\Gamma_{b} \widetilde{\Omega}_{a b}^{2}\right)\right. \\
& \left.+\frac{1}{q_{a}}\left[4 \delta\left(\delta \delta_{1}-\tilde{\Omega}_{a b}^{2}\right)+\delta_{1} \Gamma_{b}^{2}\right]\right] /\left[\left(\delta \delta_{1}-\frac{\Gamma_{a} \Gamma_{b}}{4}\right.\right. \\
& \left.\left.-\widetilde{\Omega}_{a b}^{2}\right)^{2}+\frac{\left(\delta \Gamma_{a}+\delta_{1} \Gamma_{b}\right)^{2}}{4}\right] .
\end{aligned}
$$

In fact, as we shall discuss in Sec. IV, the difference in the $\mathrm{Li}^{+}$yield $Y$, as obtained from the numerical integration of Eq. (5), and as obtained from

$$
Y=R \tau
$$

where $\tau$ is the full width at half maximum of the synchrotron pulse, is hardly visible in the figures to be presented.

\section{ATOMIC STRUCTURE AND PARAMETERS}

The atomic structure and parameters entering the model represent a major task, and have been obtained through fully correlated atomic structure calculations based on the complex-scaling method, in combination with a discretized basis constructed in terms of $B$ splines $[25,15,16]$. The calculations provide energies and widths, as well as the wave functions needed for the calculation of the dipole matrix elements. For given total angular momentum, spin, and parity, the three-electron eigenstates and eigenenergies are obtained by diagonalizing the complex rotated atomic Hamiltonian (see, e.g., Ref. [30], and references therein) in a basis spanned by three-electron basis functions, constructed by coupling the angular momenta and spins of the three electrons, and are expressed in terms of antisymmetrized linear combinations of products of one-electron orbitals.

\section{A. Structure}

The complex rotation transformation $r \rightarrow r \exp (i \theta)$ gives the three-electron Hamiltonian

$$
H(\theta)=\sum_{j=1}^{3} h_{j}(\theta)+e^{-i \theta} \sum_{j<k} \frac{1}{r_{j k}}
$$

with $r_{j k}=\left|\vec{r}_{j}-\vec{r}_{k}\right|$ and

$$
h_{j}(\theta)=-\frac{1}{2} \nabla_{r_{j}}^{2} e^{-2 i \theta}-\frac{1}{r_{j}} e^{-i \theta},
$$

where the indices $(j, k)$ run over the three electrons. The complex-rotation method allows us to represent the continuum of the outgoing (autoionized or laser-ionized) electrons in a limited box, and simultaneously reveals the triply excited states through the complex energy $E=E^{r}-i \Gamma / 2$, where $E^{r}$ is the position of the resonance and $\Gamma$ its width.

The one-electron orbitals are expanded in terms of spherical harmonics

$$
\phi_{n l m}(\vec{r})=\frac{P_{n l}(r)}{r} Y_{l m}(\Omega)
$$

and the radial parts $P_{n l}\left(r_{j}\right)=r R_{n l}\left(r_{j}\right)$,

$$
\left[\frac{e^{-2 i \theta}}{2} \frac{d^{2}}{d r_{j}^{2}}+E+\frac{e^{-i \theta}}{r_{j}}-\frac{e^{-i 2 \theta} l(l+1)}{2 r_{j}^{2}}\right] P_{n l}\left(r_{j}\right)=0,
$$


TABLE I. Couplings all expressed in atomic units. The atomic unit of intensity is $3.51 \times 10^{16} \mathrm{~W} / \mathrm{cm}^{2}$.

\begin{tabular}{ccccccc}
\hline \hline$q_{a}$ & $q_{a b}$ & $\Gamma_{a}$ & $\Gamma_{b}$ & $\tilde{\Omega}_{g a}$ & $\tilde{\Omega}_{a b}$ & $\gamma_{g}$ \\
\hline-2.9 & 48 & $4.83 \times 10^{-3}$ & $3.06 \times 10^{-3}$ & $0.0091 F_{1}(t) / 2$ & $2.1 F_{2}(t) / 2$ & $0.06 I_{1}(t) / 4$ \\
\hline \hline
\end{tabular}

with negative and positive eigenvalues, are subsequently expanded in terms of a number $N$ of $B$ splines, of order $k$ within a spherical box $[0 ; R]$

$$
P_{n l}\left(r_{j}\right)=\sum_{i}^{N} c_{i}^{n l} B_{i}^{k}\left(r_{j}\right)
$$

defined on a sinusoidal grid. Here $B_{i}^{k}\left(r_{j}\right)$ is the $i$ th $B$ spline of order $k$, a polynomial of order $k-1$ [31]. The boundary condition is $P_{n l}(0)=P_{n l}(R)=0$, and the $c_{i}^{n l}$, s are determined by matrix diagonalization. The radius of the box has to be chosen judiciously for the physical problem at hand. Obviously, we need to be able to describe the high degree of correlation between three intrashell electrons. The demand on correlation, and the fact that each electron in the triply excited state on the average "sees" a larger nuclear charge than if the $K$ shell were not empty, favors a rather small box, and indeed we have obtained good results with $R$ $=(15-25) a_{0}$. Although, in principle, other ways of implementation with $B$ splines can be contemplated, in practice it is essential to have one-electron orbitals available, as this allows a detailed choice of configurations for the final diagonalization, which makes the problem tractable with respect to computer memory and CPU time. We note that a similar complex-scaling and $B$-spline approach was recently used to determine triply excited resonances in $\mathrm{He}^{-}$[25] and also in Li [15].

With the one-electron orbitals at hand, the antisymmetric three-electron basis functions are constructed by standard coupling of angular momenta [32]. The lower eigenstates represent bound states of the system, and those lying above the ionization threshold provide a discretized representation of the continuum. The complex scaling angle $\theta=0.2$ and the basis were chosen so as to represent sufficiently accurately the triply excited states as to well as the dominant decay channels with $1 s 2 s$ and $1 s 2 p$ cores. For the $2 s^{2} 2 p\left({ }^{2} P^{o}\right)$ resonance, with 501 three-electron basis states, we obtain $E_{a}^{r}=-2.24$ a.u. and $\Gamma_{a}=131 \mathrm{meV}$, and for the $2 s 2 p^{2}\left({ }^{2} D^{e}\right)$, with 520 basis states, we obtain $E_{b}^{r}=$ -2.14 a.u. and $\Gamma_{b}=83 \mathrm{meV}$, which both compare well with benchmark [17] and other theoretical values [6]. For the ground state we find an energy of $\sim-7.44$ a.u. (to be compared with the table value of $\sim 7.47$ a.u.) with bases ranging from $\sim 300$ to 900 in size. For these calculations we used $200 B$ splines of order $k=5$ in a box of $R=15 a_{0}$.

\section{B. Parameters}

As described above, the complex scaling method allows for a rather straightforward numerical implementation of the calculation of resonance energies and widths and the corresponding eigenstates. With the eigenfunctions at hand, the dipole matrix element between the $1 s^{2} 2 s\left({ }^{2} S^{e}\right)$ ground state and the lower triply excited state $2 s^{2} 2 p\left({ }^{2} P^{o}\right)$ is readily calculated, and is conveniently written as a sum of its real and imaginary parts

$$
D_{g a}^{(r e)}+i D_{g a}^{(i m)}
$$

which we may rewrite as

$$
D_{g a}^{(r e)}\left(1-\frac{i}{q_{a}}\right)=\left\langle\bar{g}_{\theta}|D| a_{\theta,\left(E_{g}+\omega_{1}\right)}^{(R)}\right\rangle,
$$

with

$$
q_{a}=-\frac{D_{g a}^{(r e)}}{D_{g a}^{(i m)}}
$$

the Fano asymmetry parameter [27] and $\left|a_{\theta,\left(E_{g}+\omega_{1}\right)}^{(R)}\right\rangle$ the complex scaled wave function pertaining to the triply excited $2 s^{2} 2 p\left({ }^{2} P^{o}\right)$ state. The inner product of the complex scaled eigenfunctions is defined in terms of the ket and the bra $\left(\left\langle\bar{g}_{\theta}\right|\right)$ where, in the latter case, the angular parts are subject to the usual complex conjugation whereas the radial parts are not. A more detailed discussion of the resonance parameters in connection with complex scaling calculations can be found, for example, in the work of Lindroth [33], and in the review of Rost et al. [34]. We also refer to Appendix B of the present paper.

Our value for the dipole matrix element $D_{g a}^{(r e)}$ $=0.0091$ a.u. is in good agreement with the value 0.0117 a.u. extracted from the oscillator strength [35]. Also, our value of $q_{a}=-2.9$ is in reasonable agreement with published results (-2.2) [1-3]. Table I summarizes our results for the couplings entering Eq. (5). The field strength is the square root of the intensity in atomic units, $F_{i}(t)=\sqrt{I_{i}(t)}$ (the atomic unit of intensity is $I_{0}=3.51 \times 10^{16} \mathrm{~W} / \mathrm{cm}^{2}$ ) and

$$
F_{i}(t)=F_{i} f_{i}(t),
$$

with $F_{i}$ the maximal field strength and $f_{i}(t)$ the pulse envelope which we assume to be of $\sin ^{2}$ type:

$$
f_{i}(t)=\left\{\begin{array}{l}
\sin ^{2}\left(\frac{\pi t}{\frac{8}{3} \tau_{i}}\right) \quad \text { for } 0<t<\frac{8}{3} \tau_{i} \\
0 \quad \text { otherwise. }
\end{array}\right.
$$

Here the prefactor of $8 / 3$ ensures that the time integral over the intensity of the light source equals the peak intensity times $\tau_{i}$, hence $\tau_{i}$ corresponds to the full width at half maximum of the temporal envelope of the intensity ( $i=1$ and 2). If the laser pulse is much longer than the synchrotron one, 
TABLE II. Energies and widths for some higher-lying triply excited states of ${ }^{2} P^{o}$ symmetry. The basis consists of 7507 three-electron states. The box radius is $R=25 a_{0}$. The number of $B$ splines is 100 , and the order is $k=9$. The complex scaling angle is $\theta=0.2$. The energies are in atomic units (a.u.) and the widths are in meV. For comparison we show values from Ref. [17] (lines 1 and 2), Ref. [18] (lines 3-6), and Ref. [9] (last line). The term energies with respect to the $1 s^{2} 2 s\left({ }^{2} S^{e}\right)$ ground state can be calculated by using -7.478678 a.u. for the ground-state energy [36].

\begin{tabular}{llccc}
\hline \hline Resonances & $E^{r}$ (a.u.) & $\begin{array}{c}E^{r} \text { (a.u.) } \\
\text { Refs. }[17,18,9]\end{array}$ & $\Gamma(\mathrm{meV})$ & $\begin{array}{c}\Gamma(\mathrm{meV}) \\
\text { Refs. }[17,18,9]\end{array}$ \\
\hline $2 s^{2} 2 p\left({ }^{2} P^{o}\right)$ & -2.247 & -2.2502755 & 146 & 117.147 \\
$2 p^{3}\left({ }^{2} P^{o}\right)$ & -2.008 & -2.0124242 & 52.7 & 52.14 \\
$2 s^{2} 3 p\left({ }^{2} P^{o}\right)$ & -1.991 & -1.993729 & 131 & 113.58 \\
{$\left[(2 s 2 p)^{3} P, 3 s\right]^{2} P^{o}$} & -1.970 & -1.971507 & 35.2 & 38.80 \\
{$\left[(2 s 2 p)^{1} P, 3 s\right]^{2} P^{o}$} & -1.875 & -1.875691 & 98.3 & 105.5 \\
{$\left[(2 p 2 p)^{3} P, 3 p\right]^{2} P^{o}$} & -1.864 & -1.865051 & 61.8 & 111.0 \\
{$\left[(2 p 2 p)^{3} P, 4 p\right]^{2} P^{o}$} & -1.833 & -1.834557 & 106 & 49.7 \\
$3 s^{2} 3 p\left({ }^{2} P^{o}\right)$ & -1.0427 & -1.043414 & 281 & 282 \\
\hline \hline
\end{tabular}

$F_{2}(t)$ can be assumed to be constant. The synchrotron intensity being sufficiently weak to place the transition in the perturbative regime implies that the same degree of ionization can be obtained at a lower intensity by making the pulse longer, and vice versa. In our calculations, we assume that the $\mathrm{Li}$ atoms interact with the light sources for the full duration of the laser pulse, i.e., from $t=0$ until $t=\frac{8}{3} \tau_{1}$. In crossed-beam experiments the actual situation might be different, as the transit time of the atoms through the light beams might be shorter than $\frac{8}{3} \tau_{i}$.

The ionization width to the nonresonant background, $\gamma_{g}$, is calculated via the spectral decomposition of the Green's function, as is standard in complex scaling calculations of the photoabsorption cross section [34], the difference here being that the lower triply excited state is excluded from the intermediate summation. For completeness, here we include the most important formulas and arguments in the derivation. From the definition of $\gamma_{g}$ of Eq. (A8a), we have

$$
\gamma_{g}(t)=2 \pi \sum_{j}\left|\Omega_{g E_{a, j}}\right|^{2}=\frac{\pi}{2} f_{1}(t)^{2} \sum_{j}\left|D_{g E_{a, j}}\right|^{2} .
$$

The problem of calculating transition matrix elements at a specific final-state energy may be reformulated by noting that (i) the modulus squared of the dipole matrix element is a real quantity, so we may use the dispersion relation

$$
\begin{aligned}
\left|D_{g, E_{j}}\right|^{2} & =\left\langle g\left|D^{\dagger}\right| E_{j}\right\rangle\left\langle E_{j}|D| g\right\rangle \\
& =\frac{1}{\pi} \operatorname{Im}\left[\lim _{\epsilon \rightarrow 0^{+}} \int d E_{j}^{\prime} \frac{\left\langle g\left|D^{\dagger}\right| E_{j}^{\prime}\right\rangle\left\langle E_{j}^{\prime}|D| g\right\rangle}{E_{j}-E_{j}^{\prime}+i \epsilon}\right]
\end{aligned}
$$

and (ii) the bound-state part of the Green's operator does not contribute to the imaginary part in Eq. (20). Hence the full spectral decomposition may be inserted and we obtain

$$
\gamma_{g}=\frac{1}{2}\left|f_{1}(t)\right|^{2} \operatorname{Im}\left\langle g\left|D^{\dagger} G^{\prime}(E) D\right| g\right\rangle
$$

The method of complex scaling provides the appropriate discretization of the continuum and in terms of complex scaled quantities [Eq. (21)] reads

$$
\begin{aligned}
\gamma_{g} & =\frac{1}{2}\left|f_{1}(t)\right|^{2} \operatorname{Im}\left\langle\bar{g}_{\theta}\left|D^{\dagger} G_{\theta}^{\prime}\left(E_{g}^{\theta}+\omega_{1}\right) D\right| g_{\theta}\right\rangle \\
& =\frac{1}{2}\left|f_{1}(t)\right|^{2} \operatorname{Im}\left[\sum_{i}{ }^{\prime} \frac{\left\langle\bar{g}_{\theta}\left|D^{\dagger}\right| i_{\theta}\right\rangle\left\langle\bar{i}_{\theta}|D| g_{\theta}\right\rangle}{E_{i}^{\theta}-E_{g}^{\theta}-\omega_{1}}\right],
\end{aligned}
$$

where, in the second line, we have introduced the full set $\left\{i_{\theta}\right\}$ of complex scaled eigenstates of ${ }^{2} P^{o}$ symmetry. The prime on the Greens function in Eqs. (21) and (22) and on the summation sign in Eq. (22) indicates that the $2 s^{2} 2 p\left({ }^{2} P^{o}\right)$ triply excited state is excluded from the summation. We have checked by comparing with the full summation (the full ionization width) and extrapolating across the resonance of the triply excited state, that this is a welljustified procedure. Again, the index $\theta$ refers to complex scaled quantities.

For laser-induced coupling between two triply excited states, we need to consider

$$
\tilde{D}_{a b}\left(1-\frac{i}{q_{a b}}\right)
$$

which, with the help of Appendix B, is identified with

$$
\left\langle\bar{a}_{\theta,\left(E_{g}+\omega_{1}\right)}^{(R)}|D| b_{\theta,\left(E_{g}+\omega_{1}+\omega_{2}\right)}^{(R)}\right\rangle,
$$

which is the dipole matrix element between the complex scaled eigenstates belonging to the $2 s^{2} 2 p\left({ }^{2} P^{o}\right) \quad(|a\rangle)$ and $2 s 2 p^{2}\left({ }^{2} D^{e}\right)(|b\rangle)$ triply excited states of Li.

\section{Comparison with existing data}

To illustrate the quality of our results, in Table II we present energies and widths for higher-lying triply excited states of ${ }^{2} P^{o}$ symmetry. For comparison we also show results obtained through benchmark saddle-point complex- 
scaling calculations by Chung and Gou $[17,18]$ (also see Ref. [9]). It is encouraging to note that our computational approach, performing just one matrix diagonalization, produces all the data on the triply excited states shown in Table II, and that the agreement with the data of Refs. $[17,18,9]$ is generally very good: We do not sacrifice accuracy at the expense of being able to calculate the atomic parameters needed for the time-dependent calculations.

For further illustration and later reference, we consider the triply excited "double hollow" $3 s^{2} 3 p\left({ }^{2} P^{o}\right)$ state in more detail, and study its coupling with the $1 s^{2} 2 s\left({ }^{2} S^{e}\right)$ ground state. In a box of radius $R=20 a_{0}$, with $50 B$ splines of order $k=9$ and a complex scaling angle of $\theta=0.2$, with 1210 basis functions we find the ground-state energy $E_{1 s^{2} 2 s\left({ }^{2} s^{e}\right)}=-7.45$ a.u., which should be compared with the table value of -7.47868 a.u. [36]. For the triply excited $3 s^{2} 3 p\left({ }^{2} P^{o}\right)$ state we find $E_{3 s^{2} 3 p\left({ }^{2} P^{o}\right)}=-1.043$ a.u. (term energy $175.12 \mathrm{eV})$ and $\Gamma_{3 s^{2} 3 p^{\left(2 P^{o}\right)}}=276 \mathrm{meV}$. The measured values for the $3 s^{2} 3 p\left({ }^{2} P^{o}\right)$ resonance are 175.165 $\pm 0.050 \mathrm{eV}$ for the term energy and $0.25 \pm 0.05 \mathrm{eV}$ for the width [9]. Azuma et al. [4] measured the $3 s^{2} 3 p\left({ }^{2} P^{o}\right)$ resonance in the $\mathrm{Li}^{2+}$ photoion channel, and found $E^{r}=175.25$ $\pm 0.10 \mathrm{eV}$ and $\Gamma=0.32 \pm 0.1$. Hence our values for the resonance parameters agree rather well with the values reported in existing measurements and calculations (see Table II). For the dipole matrix element between the $1 s^{2} 2 s\left({ }^{2} S^{e}\right)$ and the $3 s^{2} 3 p\left({ }^{2} P^{o}\right)$ states, we find $\widetilde{D}_{g a}=8.6 \times 10^{-4} a_{0}$. And for the $q$ value we obtain $q=3.4$. We note, that Azuma et al. [4] also reported a $q$ value, $q=2.1 \pm 0.1$, for the resonance. However, these two $q$ values cannot be directly compared, since our value $\left(q_{+}\right)$is related to the interaction with the one-electron continuum (states of $\mathrm{Li}^{+}$) whereas the value of Azuma et al. $\left(q_{2+}\right)$ is related to the two-electron continuum (the $\mathrm{Li}^{2+}$ channel).

\section{RESULTS}

Figures 2 and 3 show the $\mathrm{Li}^{+}$photoion yield as a function of the detunings of the light sources for a series of intensities and detunings, as detailed in the captions. In all cases studied, we see how the line shapes change drastically with the intensity of the laser coupling the $2 s^{2} 2 p\left({ }^{2} P^{o}\right)$ and $2 s 2 p^{2}\left({ }^{2} D^{e}\right)$ triply excited states. At the lowest intensity we have a line shape corresponding to the absence of a coupling between two triply excited states. As the intensity is increased, a double-peak structure builds up in Fig. 2, while we observe a window resonance in Fig. 3. The double-peak structure in Fig. 2 is due to the ac Stark splitting as a result of the laser-induced oscillation between $|a\rangle$ and $|b\rangle$. At the highest intensity in Fig. 2(a), the separation between the two peaks is $\sim 2.8 \Gamma_{a}$ and also equal to twice the Rabi frequency, $2 \widetilde{\Omega}_{a b}$. Hence the photoion spectrum carries direct information about the dipole matrix element between the triply excited states. In Fig. 2(b), the splitting at the largest intensity is approximately equal to twice the generalized Rabi frequency, $2 \sqrt{\widetilde{\Omega}_{a b}^{2}+\left(\delta_{2} / 2\right)^{2}}$. In Fig. 2(a) we have chosen $\delta_{2}$ $=0$, and in Fig. 2(b) $\delta_{2}=\Gamma_{a}$, and we see how, e.g., the line shape at $I_{1}=1.4 \times 10^{11} \mathrm{~W} / \mathrm{cm}^{2}$ is significantly different in

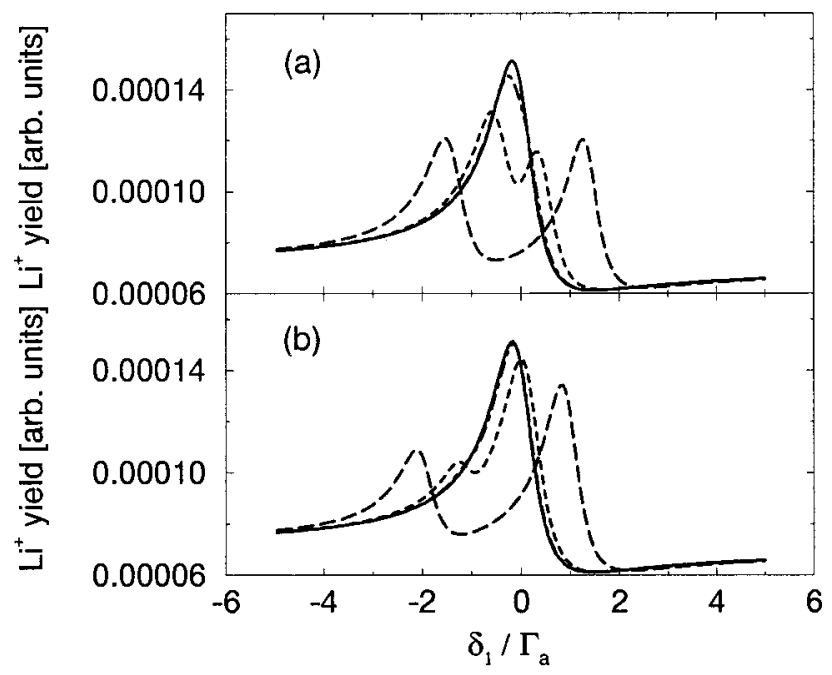

FIG. 2. $\mathrm{Li}^{+}$photoion yield, as a function of the detuning $\delta_{1}$, associated with the transition between the ground state and the $2 s^{2} 2 p\left({ }^{2} P^{o}\right)$ triply excited state. The laser coupling the $2 s^{2} 2 p\left({ }^{2} P^{o}\right)$ and $2 s 2 p^{2}\left({ }^{2} D^{e}\right)$ states is (a) on resonance with the transition $\left(\delta_{2}\right.$ $=0$ ), and (b) detuned off resonance by the width of the $2 s^{2} 2 p\left({ }^{2} P^{o}\right)$ state $\left(\delta_{2}=\Gamma_{a}\right)$. The full, dot-dashed, dashed, and long-dashed curves correspond to $I_{2}=1.4 \times 10^{7}, 1.4 \times 10^{10}, 1.4$ $\times 10^{11}$, and $1.4 \times 10^{12} \mathrm{~W} / \mathrm{cm}^{2}$, respectively. The intensity of the $\tau_{1}=10 \mathrm{ps}$ synchrotron pulse is $I_{1}=4 \times 10^{8} \mathrm{~W} / \mathrm{cm}^{2}$. We clearly see that the laser coupling induces an ac Stark splitting of the line, the peak separation of which equals twice the Rabi frequency.

the two cases. The difference in height of the peaks in the structures of Fig. 2(a), and the asymmetry in the position of the minimum with respect to $\delta_{1}=0$ is due to the finite value of $q_{a}$, i.e., due to the slight asymmetry in the line shape of the triply excited $2 s^{2} 2 p\left({ }^{2} P^{o}\right)$ resonance (in the absence of

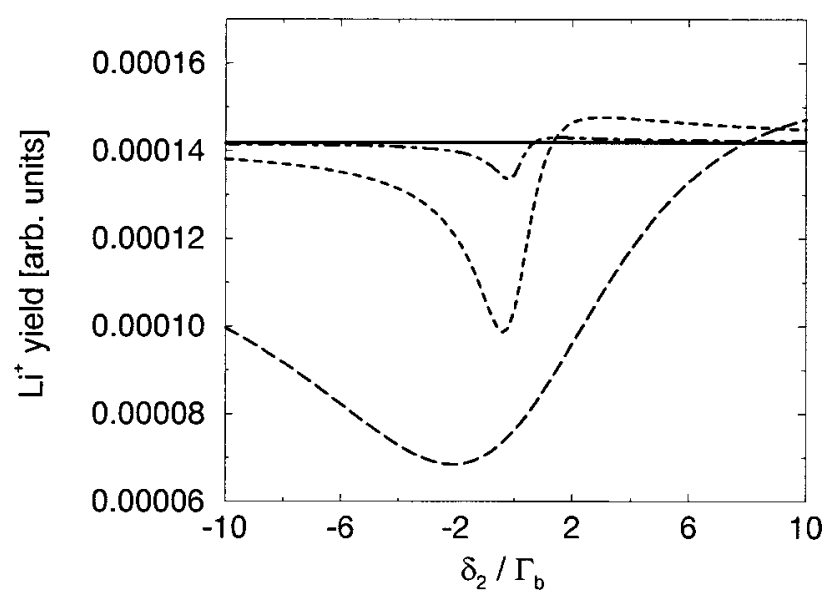

FIG. 3. $\mathrm{Li}^{+}$photoion yield as a function of the detuning $\delta_{2}$, associated with the $2 s^{2} 2 p\left({ }^{2} P^{o}\right) \rightarrow 2 s 2 p^{2}\left({ }^{2} D^{e}\right)$ transition. The synchrotron coupling the ground state with the $2 s^{2} 2 p\left({ }^{2} P^{o}\right)$ triply excited state is on resonance $\left(\delta_{1}=0\right)$. The full, dot-dashed, dashed, and long-dashed curves correspond to $I_{2}=1.4 \times 10^{6}, 1.4 \times 10^{10}$, $1.4 \times 10^{11}, 1.4 \times 10^{12} \mathrm{~W} / \mathrm{cm}^{2}$, respectively. The intensity of the $\tau_{1}$ $=10$ ps synchrotron pulse is $I_{1}=4 \times 10^{8} \mathrm{~W} / \mathrm{cm}^{2}$. With increasing laser intensity, the triply excited states become coupled, and a window resonance appears. 


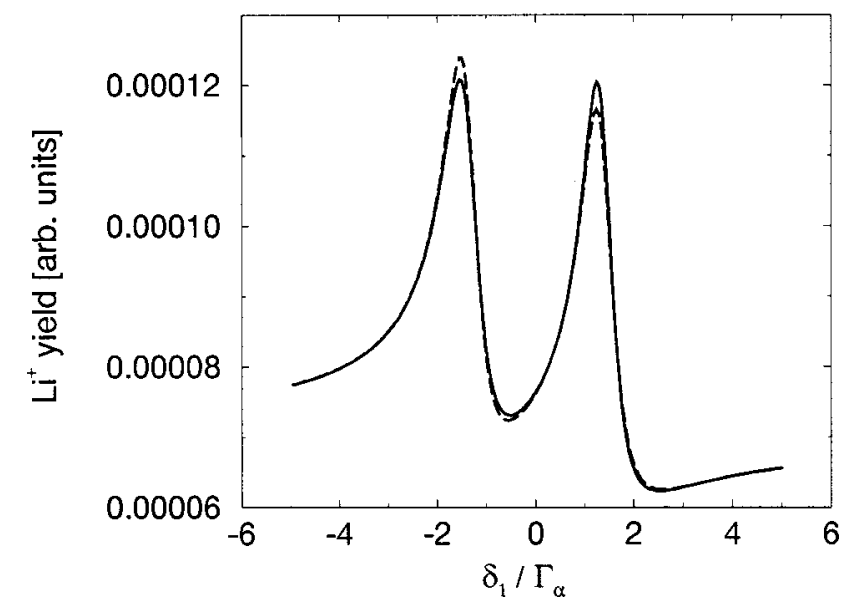

FIG. 4. $\mathrm{Li}^{+}$photoion yield as a function of the detuning $\delta_{1}$, associated with the transition between the ground state and the $2 s^{2} 2 p\left({ }^{2} P^{o}\right)$ triply excited state. The laser coupling the $2 s^{2} 2 p\left({ }^{2} P^{o}\right)$ and $2 s 2 p^{2}\left({ }^{2} D^{e}\right)$ states is on resonance with the transition $\left(\delta_{2}\right.$ $=0$ ), and its intensity is $I_{2}=1.4 \times 10^{12} \mathrm{~W} / \mathrm{cm}^{2}$. The intensity of the $\tau_{1}=10$ ps synchrotron pulse is $I_{1}=4 \times 10^{8} \mathrm{~W} / \mathrm{cm}^{2}$. The full curve is the result of a numerical integration of Eq. (5), while the dashed curve pertains to the analytical yield $Y=R \tau$ given by Eq. (8), with $R$ given by Eq. (7b) and $\tau=10$ ps. The curves differ only in the relative heights of the peaks.

the laser). In Fig. 2(b) this difference is enhanced by asymmetric off-resonant excitation.

In Fig. 3 the frequency $\omega_{1}$ is on resonance $\left(\delta_{1}=0\right)$, while the laser frequency $\omega_{2}$ is varied. At the lowest intensity, no coupling to the $2 s 2 p^{2}\left({ }^{2} D^{e}\right)$ triply excited state occurs, as demonstrated by the straight line in the figure. With increasing intensity, the triply excited states become coupled, and a window resonance appears. We note that the minimum in the window moves to the left as the laser intensity is increased due to ac Stark splitting. We know of no other way that such coupling between triply excited states, or equivalent transitions exploring the highly correlated manifold of the fourbody Coulomb problem, can be studied.

We note that the analytical formula of Eq. (7b) for the photoion rate $R$ accounts for the variations in Figs. 2 and 3 in the weak-probe limit $\left(\widetilde{\Omega}_{g a} \ll 1\right)$. In fact, the difference in the $\mathrm{Li}^{+}$yield as obtained from the numerical integration of Eq. (5) and as obtained from Eq. (8) is hardly visible in Figs. 2 and 3. Figure 4 gives a representative example. We see that the results of the numerical integration of Eq. (5) and from the yield as given by Eq. (8) for the appropriate pulse parameters as detailed in the caption, do only differ in the relative heights of the peaks. As seen from Fig. 2 and as discussed in detail above, the distortion of the line shape evolves into a double-peak structure at sufficiently high laser intensity. A careful examination of the extrema of $R$ shows that the ac stark splitting is given by $2 \times \widetilde{\Omega}_{a b}$ for $\delta_{2}=0$, i.e., when the laser coupling the two triply excited states is on resonance. For $\delta_{2}=\Gamma_{a}$ the ac Stark splitting involves a much more complicated expression, but is still approximately equal to twice the generalized Rabi frequency, $2 \times \sqrt{\widetilde{\Omega}_{a b}^{2}+\left(\delta_{2} / 2\right)^{2}}$. These results are most easily realized by rewriting the denominator of Eq. (7b) as

$$
\left(\frac{\left(\delta_{1}+\delta_{2}\right) \delta_{1}}{\widetilde{\Omega}_{a b}^{2}}-1\right)^{2} \widetilde{\Omega}_{a b}^{4}+\frac{\left[\left(\delta_{1}+\delta_{2}\right) \Gamma_{a}+\delta_{1} \Gamma_{b}\right]^{2}}{4},
$$

which is valid when we have a splitting of the order of $\Gamma_{a}$ or larger, i.e., when $\Gamma_{a} \Gamma_{b} / 4 \widetilde{\Omega}_{a b}^{2} \ll 1$ (which is certainly the case of the largest intensities in Fig. 2). In this regime, the splitting is given by the difference in the roots of the first term of Eq. (25), and the result $2 \times \sqrt{\widetilde{\Omega}_{a b}^{2}+\left(\delta_{2} / 2\right)^{2}}$ is readily obtained. In the case of $\delta_{1}=0$, pertaining to Fig. 3, it is seen from Eq. (7b), that it is the sign of $\delta_{2} / q_{a}$ which determines the decrease/increase as a function of $\delta_{2}$ for increasing laser intensity: For detunings to the red the $\mathrm{Li}^{+}$yield decreases compared to the laser-free situation for negative $q_{a}$, and vice versa, for positive $q_{a}$.

\section{CONCLUSION AND OUTLOOK}

In conclusion, we have presented results on stimulated transitions between highly correlated triply excited states in a fundamental system. Our findings point to a unique tool, readily implementable with present synchrotron and laser technology, for the exploration of manifolds of triply excited states and the unusual correlation properties they entail. Not only can couplings between such states be thus probed, but also selective study of decays into single- or double-electron breakup can be pursued for states lying in energy above the first doubly excited state of $\mathrm{Li}^{+}$. For example, in view of the presented results, we can realistically contemplate the control, in a channel specific-way, of the decays of triply excited states above $151.7 \mathrm{eV}$ where the phenomenon of two-step double autoionization from triply excited Li via doubly excited $\mathrm{Li}^{+}$to $\mathrm{Li}^{2+}$ becomes possible [11]. Specifically, the excitation of a state like $2 s 2 p\left({ }^{1} P\right) 3 s\left({ }^{2} P^{o}\right)$ from the ground state in the presence of a laser coupling it to an even-parity state [like, e.g., $\left.2 s 2 p^{2}\left({ }^{2} D^{e}\right)\right]$ which decays predominantly to $\mathrm{Li}^{+}$would single out that route of decay of the initially excited resonance, instead of the multiple cascade that it would otherwise follow. The requirements on pulse durations and intensities for laser and synchrotron sources are well within the range of recent and ongoing experiments on such states. A further calibration of the possibilities is provided by observations of analogous transitions (at much lower excitation energies) between doubly excited states in a two-electron system $(\mathrm{Mg})$ [37].

\section{ACKNOWLEDGMENTS}

L.B.M. acknowledges support of the Danish Natural Science Research Council (Grant No. 9800755). P.S. acknowledges support of the Feodor Lynen-Stipendium (V-3FLF106691) from the Alexander von Humboldt-Stiftung. LPTMS is a unité de recherche de l'Université Paris XI associée au CNRS. 


\section{APPENDIX A}

This appendix gives explicit expressions for the atomic parameters entering Eqs. (3)-(5). Due to the vanishing continuum-continuum couplings the contributions from the different continua add incoherently, as shown in the formulas to follow.

The laser-induced shift of the ground state is

$$
S_{g}=\sum_{j} S_{g, j}
$$

with

$$
S_{g, j}=\mathrm{P} \int d E_{a, j} \frac{\left|\Omega_{g E_{a, j}}(t)\right|^{2}}{E_{g}+\omega_{1}-E_{a, j}} .
$$

The light-induced shift of $|a\rangle$ is

$$
S_{a}=\sum_{k} S_{a, k}
$$

with

$$
S_{a, k}=\mathrm{P} \int d E_{b, k} \frac{\left|\Omega_{a E_{b, k}}\right|^{2}}{E_{g}+\omega_{1}+\omega_{2}-E_{b, k}} .
$$

The light-induced shift of $|b\rangle$ is

$$
S_{b}=\sum_{j} S_{b, j}
$$

with

$$
S_{b, j}=\mathrm{P} \int d E_{a, j} \frac{\left|\Omega_{b E_{a, j}}\right|^{2}}{E_{g}+\omega_{1}-E_{a, j}} .
$$

The laser-induced shift due to the two-photon coupling of $|g\rangle$ and $|b\rangle$, via the nonresonant part of the continuum belonging to the autoionizing states with discrete part $|a\rangle$, is

$$
S_{g b}=\sum_{j} S_{g b, j}
$$

with

$$
S_{g b, j}=\mathrm{P} \int d E_{a, j} \frac{\Omega_{g E_{a, j}} \Omega_{E a, j b}}{E_{g}+\omega_{1}-E_{a, j}} .
$$

The positions of the resonances are

$$
E_{s}^{r}=E_{s}+F_{s}, \quad s=a, b,
$$

where the self-energy shifts are

$$
F_{s}=\sum_{i} F_{s, i}
$$

with

$$
F_{s, i}=\mathrm{P} \int d E_{s, i} \frac{\left|V_{s E_{s, i}}\right|^{2}}{E_{g}+\omega_{s}-E_{s, i}},
$$

and $\omega_{s}=\omega_{1}$ for $s=a$ while $\omega_{s}=\omega_{1}+\omega_{2}$ for $s=b$. The autoionization widths are

$$
\Gamma_{s}=\sum_{i} \Gamma_{s, i}, \quad s=a, b,
$$

with

$$
\Gamma_{s, i}=2 \pi\left|V_{s E_{s, i}}\right|^{2}
$$

and $E_{s, i}=E_{g}+\omega_{s}$.

The ionization width of $|g\rangle$ to the nonresonant part of the continuum belonging to the autoionizing state, with a discrete part given by $|a\rangle$, is

$$
\gamma_{g}=\sum_{j} \gamma_{g, j}
$$

with

$$
\gamma_{g, j}=2 \pi\left|\Omega_{g, E_{a, j}}\right|^{2},
$$

and $E_{a, j}=E_{g}+\omega_{1}$. The ionization width of $|a\rangle$ to the nonresonant background of the autoionizing state, with a discrete part $|b\rangle$, is

$$
\gamma_{a}=\sum_{k} \gamma_{a, k}
$$

with

$$
\gamma_{a, k}=2 \pi\left|\Omega_{a E_{b, k}}\right|^{2},
$$

and $E_{b, k}=E_{g}+\omega_{1}+\omega_{2}$

The ionization width of $|b\rangle$ to the nonresonant background of the autoionizing state with discrete part $|a\rangle$ is

$$
\gamma_{b}=\sum_{j} \gamma_{b, j}
$$

with

$$
\gamma_{b, j}=2 \pi\left|\Omega_{b E_{a, j}}\right|^{2},
$$

and $E_{a, j}=E_{g}+\omega_{1}$ The corresponding ionization width into the nonresonant continuum due to the coupling of $|g\rangle$ and $|b\rangle$, via $\left|E_{a, j}\right\rangle$, is

$$
\gamma_{g b}=\sum_{j} \gamma_{g b, j}
$$

with

$$
\gamma_{g b, j}=2 \pi \Omega_{g E_{a, j}} \Omega_{E_{a, j} b},
$$

and $E_{a, j}=E_{g}+\omega_{1}$.

The Rabi frequency of the $|g\rangle \rightarrow|a\rangle$ transition is 


$$
\tilde{\Omega}_{g a}=\Omega_{g a}+\mathrm{P} \sum_{j} \int d E_{a, j} \frac{\Omega_{g E_{a, j}} V_{E_{a, j} a}}{E_{g}+\omega_{1}-E_{g}} .
$$

The generalized Rabi frequency of the $|a\rangle \rightarrow|b\rangle$ transition is

$$
\begin{aligned}
\tilde{\Omega}_{a b}= & \Omega_{a b}+\mathrm{P} \sum_{j} \int d E_{a, j} \frac{V_{a E_{a, j}} \Omega_{E_{a, j} b}}{E_{g}+\omega_{1}-E_{a, j}} \\
& +\mathrm{P} \sum_{k} \int d E_{b, k} \frac{\Omega_{a E_{b, k}} V_{E_{b, k} b}}{E_{g}+\omega_{1}+\omega_{2}-E_{b, k}} .
\end{aligned}
$$

The Fano parameter [27] belonging to the autoionizing state with discrete part $|a\rangle$ is

$$
q_{a}=\frac{\tilde{\Omega}_{g a}}{\pi\left(\sum_{j} \Omega_{g E_{a, j}} V_{E_{a, j} a}\right)},
$$

with $E_{a, j}=E_{g}+\omega_{1}$. Note that, in the case of only one continuum, the simple relation

$$
q_{a}^{2}=\frac{4\left|\tilde{\Omega}_{g a}\right|^{2}}{\gamma_{g} \Gamma_{a}}
$$

follows directly from Eq. (A14) and the corresponding definitions of the widths $\Gamma_{a}, \gamma_{g}$ [see Eqs. (A7a) and (A8a), respectively]. The generalized Fano parameter for the $|a\rangle$ $\rightarrow|b\rangle$ transition is

$$
q_{a b}=\frac{\tilde{\Omega}_{a b}}{\pi\left(\sum_{j} V_{a E_{a, j}} \Omega_{E_{a, j} b}+\sum_{k} \Omega_{a E_{b, k}} V_{E_{b, k} b}\right)},
$$

where the first term in the denominator is evaluated at $E_{a, j}$ $=E_{g}+\omega_{1}$, and the second is evaluated $E_{b, k}=E_{g}+\omega_{1}+\omega_{2}$.

\section{APPENDIX B}

This appendix examines the connection between complex scaled wave functions and the atomic parameters need for the solution of Eq. (5). Here it is convenient to work directly with the dipole matrix elements.

The matrix element of interest for the coupling between the $1 s^{2} 2 s\left({ }^{2} S^{e}\right)$ ground state and the $2 s^{2} 2 p\left({ }^{2} P^{o}\right)$ triply excited state is

$$
D_{g a}^{F}=\widetilde{D}_{g a}\left(1-\frac{i}{q_{a}}\right)
$$

which, to make the connection to complex scaled quantities, is rewritten as

$$
\begin{aligned}
D_{g a}^{F} & =\left\langle g\left|\left(D+\sum_{j} \int d E_{a j} \frac{D\left|E_{a j}\right\rangle\left\langle E_{a j}\right| V}{E_{g}+\omega_{1}-E_{a j}}\right)\right| a\right\rangle \\
& =\left\langle g\left|D\left(1+\sum_{j} \int d E_{a j} \frac{\left|E_{a j}\right\rangle\left\langle E_{a j}\right| V}{E_{g}+\omega_{1}-E_{a j}}\right)\right| a\right. \\
& =\left\langle\bar{g}_{\theta}|D| a_{\theta,\left(E_{g}+\omega_{1}\right)}^{(R)}\right\rangle,
\end{aligned}
$$

with $\left|a_{\theta,\left(E_{g}+\omega_{1}\right)}^{(R)}\right\rangle$ the complex scaled wave function of the triply excited $2 s^{2} 2 p\left({ }^{2} P^{o}\right)$ state. For the coupling between two triply excited states $2 s^{2} 2 p\left({ }^{2} P^{o}\right)$ and $2 s 2 p^{2}\left({ }^{2} D^{e}\right)$, the quantity which enters in our theory is

$$
D_{a b}^{F}=\widetilde{D}_{a b}\left(1-\frac{i}{q_{a b}}\right)
$$

which, to make the connection to complex scaled quantities, is rewritten as

$$
\begin{aligned}
D_{a b}^{F}= & \langle a|\left(D+\sum_{j} \int d E_{a j} \frac{V\left|E_{a j}\right\rangle\left\langle E_{a j}\right| D}{E_{g}+\omega_{1}-E_{a j}}\right. \\
& \left.+\sum_{k} \int d E_{b k} \frac{D \mid E_{b k}\left\langle E_{b k}\right| V}{E_{g}+\omega_{1}+\omega_{2}-E_{b k}}\right)|b\rangle \\
\simeq & \langle a|\left(1+\sum_{j} \int d E_{a j} \frac{V\left|E_{a j}\right\rangle\left\langle E_{a j}\right|}{E_{g}+\omega_{1}-E_{a j}}\right) D \\
& \times\left(1+\sum_{k} \int d E_{b k} \frac{\left|E_{b k}\right\rangle\left\langle E_{b k}\right| V}{E_{g}+\omega_{1}+\omega_{2}-E_{b k}}\right)|b\rangle,
\end{aligned}
$$

where the $\simeq$ sign signals that we have effectively introduced an extra term

$$
\sum_{j, k} \int d E_{a j} d E_{b k} \frac{V\left|E_{a j}\right\rangle\left\langle E_{a j}|D| E_{b k}\right\rangle\left\langle E_{b k}\right| V}{\left(E_{g}+\omega_{1}-E_{a j}\right)\left(E_{g}+\omega_{1}+\omega_{2}-E_{b k}\right)} .
$$

The term of Eq. (B5) is expected to be very small and, in fact, consistent with our approximation neglecting continuum-continuum couplings, it is set equal to zero. From Eq. (B4) we may now make the identification

$$
D_{a b}^{F}=\left\langle\bar{a}_{\theta,\left(E_{g}+\omega_{1}\right)}^{(R)}|D| b_{\theta,\left(E_{g}+\omega_{1}+\omega_{2}\right)}^{(R)}\right\rangle,
$$

which is the dipole matrix element between the complex scaled eigenstates corresponding to the $2 s^{2} 2 p\left({ }^{2} P^{o}\right)$ and $2 s 2 p^{2}\left({ }^{2} D^{e}\right)$ triply excited states.
[1] L. M. Kiernan et al., Phys. Rev. Lett. 72, 2359 (1994).

[2] L. M. Kiernan et al., J. Phys. B 28, L161 (1995).

[3] Y. Azuma et al., Phys. Rev. Lett. 74, 3768 (1995).

[4] Y. Azuma et al., Phys. Rev. Lett. 79, 2419 (1997).

[5] L. Journel et al., Phys. Rev. Lett. 76, 30 (1996).

[6] S. Diehl et al., Phys. Rev. Lett. 76, 3915 (1996).
[7] D. Cubaynes et al., Phys. Rev. Lett. 77, 2194 (1996).

[8] S. Diehl et al., Phys. Rev. Lett. 79, 1241 (1997).

[9] S. Diehl et al., Phys. Rev. A 56, R1071 (1997).

[10] S. Diehl et al., Phys. Rev. Lett. 84, 1677 (2000).

[11] S. Diehl et al., J. Phys. B 30, L595 (1997).

[12] K. Berrington and S. Nakazaki, J. Phys. B 31, 313 (1998). 
[13] L. Vo Ky et al., Phys. Rev. A 58, 3688 (1998).

[14] M. J. Conneely and L. Lipsky, Phys. Rev. A 61, 032506 (2000).

[15] G. Verbockhaven and J. E. Hansen, Phys. Rev. Lett. 84, 2810 (2000).

[16] L.B. Madsen, P. Schlagheck, and P. Lambropoulos, Phys. Rev. Lett. 85, 42 (2000).

[17] K. T. Chung and B. C. Gou, Phys. Rev. A 52, 3669 (1995).

[18] K. T. Chung and B. C. Gou, Phys. Rev. A 53, 2189 (1996).

[19] T. Morishita and C. D. Lin, Phys. Rev. A 59, 1835 (1999).

[20] T. Morishita, Y. Li, and C. D. Lin, Phys. Rev. A 58, 4214 (1998).

[21] T. Morishita and C. D. Lin, Phys. Rev. A 57, 4268 (1998).

[22] T. Morishita, O. I. Tolstikhin, S. Watanabe, and M. Matsuzawa, Phys. Rev. A 56, 3559 (1997).

[23] X. Yang, C. G. Bao, and C. D. Lin, Phys. Rev. Lett. 76, 3096 (1996); Phys. Rev. A 53, 3934 (1996).

[24] H. L. Zhou, S. T. Manson, P. Faucher, and L. Vo Ky, Phys. Rev. A 62, 012707 (2000).
[25] N. Brandefelt and E. Lindroth, Phys. Rev. A 59, 2691 (1999).

[26] U. I. Safronova and V. S. Senashenko, J. Phys. B 11, 2623 (1978).

[27] U. Fano, Phys. Rev. 124, 1866 (1961).

[28] K. T. Chung, Phys. Rev. A 59, 2065 (1999).

[29] J. E. Hansen, J. Phys. B 8, L403 (1975).

[30] B. Junker, Adv. At. Mol. Phys. 18, 207 (1982).

[31] C. deBoor, A Practical Guide to Splines (Springer-Verlag, New York, 1978).

[32] I. I. Sobelman, Atomic Spectra and Radiative Transitions (Springer, Berlin, 1992).

[33] E. Lindroth, Phys. Rev. A 52, 2737 (1995).

[34] J. M. Rost, K. Schulz, M. Domke, and G. Kaindl, J. Phys. B 30, 4663 (1997).

[35] K. T. Chung, Phys. Rev. A 25, 1596 (1982).

[36] K. T. Chung, Phys. Rev. A 44, 5421 (1991).

[37] N. E. Karapanagioti et al., Phys. Rev. Lett. 74, 2431 (1995); Phys. Rev. A 53, 2587 (1996). 